\title{
SIMPLIFIED STRUCTURES FOR TWO-DIMENSIONAL ADAPTIVE NOTCH FILTERS
}

\author{
Soo-Chang Pei, Chang-Long Wu, and Jian-Jiun Ding \\ Department of Electrical Engineering, National Taiwan University, Taipei, Taiwan, R.O.C. \\ Email address: pei@cc.ee.ntu.edu.tw
}

\begin{abstract}
In this paper, three new methods for two-dimensional (2-D) adaptive notch filter (ANF) are proposed. They not only contain similar and simpler structures but also can be derived by simpler adaptive algorithms compared with the previous ones. They are helpful for 2-D signal processing. Simulation results show that they can eliminate 2-D sinusoidal signals successfully.
\end{abstract}

\section{INTRODUCTION}

The Adaptive notch filter (ANF) is used to remove narrowband or sinusoidal interference embedded in broadband signal. During these years, many structures and methods for onedimensional adaptive notch filters have been proposed [1][2]. For two-dimensional case, however, there haven't been many articles talking about it. Although [3]-[5] proposed several techniques for designing 2-D notch filters, and, a method was proposed in [6] for 2-D adaptive notch filter recently, their structures were not simple enough.

Here, we propose two types of structures for 2-D adaptive notch filters whose complexities and orders were lower than the ones in the former workings and have satisfying performances when tracking 2-D sinusoidal signals. First type is based on the outer product expansion [5][6] but with a few changes so as to cancel the redundant part and simplify the structure of the 2-D notch filter (NF). The other type simply was derived from the frequency-shift method, which moves a notch at zero frequency toward the desired frequencies, respectively. The NFs coming from these two methods have lower orders, and we can find new 2-D ANFs based on the proposed NFs and Burg algorithm [6][7].

This paper is organized as follows. First we express our new 2-D notch filter structures. Then, based on it, the adaptive algorithm is proposed. Finally, simulations are given to evaluate their performances.

\section{TWO-DIMENSIONAL NOTCH FIL- TERS}

The frequency response of an ideal real-coefficient 2-D NF is

$$
H_{n}\left(e^{j \omega_{1}}, e^{i \omega_{2}}\right)=\left\{\begin{array}{lc}
0, & \left(\omega_{1}, \omega_{2}\right)=\left(\omega_{1}^{\prime}, \omega_{2}^{\prime}\right) . \\
0, & \left(\omega_{1}, \omega_{2}\right)=\left(-\omega_{1}^{\prime},-\omega_{2}^{\prime}\right) . \\
1, & \text { otherwise. }
\end{array}\right.
$$

where $\left(\omega_{i}, \omega_{2}^{\prime}\right)$ is the notch frequency, and for real 2-D NF, there is another notch at $\left(-\omega_{1},-\omega_{2}^{*}\right)$.

The main method to construct the two-dimensional notch filter (2-D NF) $H_{d}\left(z_{1}, z_{2}\right)$ is subtracting the two-dimensional diagonal bandpass filter (BPF) $H_{c}\left(z_{1}, z_{2}\right)$ from (1):

$$
H_{n}\left(z_{1}, z_{2}\right)=1-H_{b}\left(z_{1}, z_{2}\right)
$$

In [5], the outer product expansion method for finding the 2-D $\mathrm{BPF}$ is given by

$$
H_{b}\left(z_{1}, z_{2}\right)=\frac{1}{2} H_{c 1}\left(z_{1}\right) H_{c 2}\left(z_{2}\right)-\frac{1}{2} H_{s 1}\left(z_{1}\right) H_{s 2}\left(z_{2}\right)
$$

where $H_{c i}\left(z_{i}\right)$ and $H_{s i}\left(z_{i}\right)$ are 1-D filters:

$$
H_{c i}\left(e^{j \omega_{1}}\right)=\left\{\begin{array}{lc}
1, & \omega_{i}=\omega_{i}^{\prime} \text { or }-\omega_{i} . \\
0, & \text { otherwise. }
\end{array}\right.
$$

$$
\text { and } \quad H_{s i}\left(e^{j \omega_{2}}\right)=\left\{\begin{array}{cc}
-j, & \omega_{i}=\omega_{i} \\
j, & \omega_{i}=-\omega_{i} \\
0, & \text { otherwise }
\end{array}\right.
$$

Furthermore, if we replace $H_{s i}\left(z_{i}\right)$ by

$$
H_{s i}\left(z_{i}\right)=H_{c i}\left(z_{i}\right) H_{a i}\left(z_{i}\right)
$$

where

$$
H_{u i}\left(e^{j \omega_{i}}\right)=\left\{\begin{array}{cc}
-j, & \omega_{i}=\omega_{i} . \\
j, & \omega_{i}=-\omega_{i} . \\
\text { anyvalue, } & \text { otherwise. }
\end{array}\right.
$$

The 2-D NFs obtained from Eqs. (2), (3) and (6) are:

$$
H_{n}\left(z_{1}, z_{2}\right)=1-\frac{1}{2} H_{c 1}\left(z_{1}\right) \cdot H_{c 2}\left(z_{2}\right) \cdot\left(1-H_{a 1}\left(z_{1}\right) \cdot H_{a 2}\left(z_{1}\right)\right)
$$


where $\boldsymbol{H}_{c i}\left(z_{i}\right)$ 's are 1-D $2^{m i}$-order BPFs and $\boldsymbol{H}_{a i}\left(z_{i}\right)$ 's are 1-D $1^{\text {st }}$-order allpass filters (APFs).

\subsection{Two-dimensional NF from simplified band- pass filters}

The following is the derivation of our type-1 NF. First consider the 1-D BPF (based on APF) in Eq.(8) to be

$$
\begin{aligned}
& H_{c i}\left(z_{i}\right)=\frac{1}{2}\left(1-\frac{r_{i}^{2}-\left(1+r_{i}^{2}\right) \cos \left(\omega_{i}^{*}\right) z_{i}^{-1}+z_{i}^{-2}}{1-\left(1+r_{i}^{2}\right) \cos \left(\omega_{i}^{\prime}\right) z_{i}^{-1}+r_{i}^{2} z_{i}^{-2}}\right) \\
& =\frac{1-r_{i}^{2}}{2} \frac{1-z_{i}^{-2}}{1-\left(1+r_{i}^{2}\right) \cos \left(\omega_{i}\right) z_{i}^{-1}+r_{i}^{2} z_{i}^{-2}}
\end{aligned}
$$

where $0<r_{i}<1$ is the pole radius. Then assume the corresponding $\boldsymbol{H}_{s i}\left(\boldsymbol{z}_{i}\right)$ 's in Eq.(3) to be

$$
H_{s i}\left(z_{i}\right)=\frac{1-r_{i}^{2}}{2} \frac{X_{i}\left(z_{i}\right)}{1-\left(1+r_{i}^{2}\right) \cos \left(\omega_{i}^{*}\right) z_{i}^{-1}+r_{i}^{2} z_{i}^{-2}}
$$

From previous discussion, we know if

$$
H_{a i}\left(e^{j \omega_{i}}\right)=\left[\frac{X_{i}\left(z_{i}\right)}{1-z_{i}^{-2}}\right]_{z_{i}=e^{j \omega i}}=-j
$$

is satisfied, the $\boldsymbol{H}_{s i}\left(z_{i}\right)$ 's in Eq.(10) will satisfy the desired frequency response in Eq.(5). After simple manipulations, we get

$$
X_{i}\left(z_{i}\right)=2 z_{i}^{-1} \sin \left(\omega_{i}^{*}\right)
$$

Therefore

$$
H_{s i}\left(z_{i}\right)=\frac{1-r_{i}^{2}}{2} \frac{2 \sin \left(\omega_{i}^{\prime}\right) z_{i}^{-1}}{1-\left(1+r_{i}^{2}\right) \cos \left(\dot{\omega}_{i}^{\prime}\right) z_{i}^{-1}+r_{i}^{2} z_{i}^{-2}}
$$

It is observed that Eq.(9) and (13) have the same denominators, and we can construct a new 2-D NF from Eq.(3), (10) and (13). Here we call this type of 2-D NFs NFI, and its block diagram is presented in Fig. 1 with a simpler structure than the conventional ones [4][5][6].

\subsection{Two-dimensional NFs by frequency-shift method}

Here we introduce a simpler method to find the 2-D NF. First we consider a 2-D BPF denoted by $H_{b, 0}\left(z_{1}, z_{2}\right)$ with only a spike at origin, that is, $\left(\omega_{1}^{\prime}, \omega_{2}^{\prime}\right)=(0,0)$. Next, we shift the spike of $H_{b, 0}\left(z_{1}, z_{2}\right)$ from $(0,0)$ to $\left(\omega_{1}^{\prime}, \omega_{2}^{\prime}\right)$ or $\left(-\omega_{1}^{\prime},-\omega_{2}^{\prime}\right)$ respectively, which means generating the 2-D BPF from:

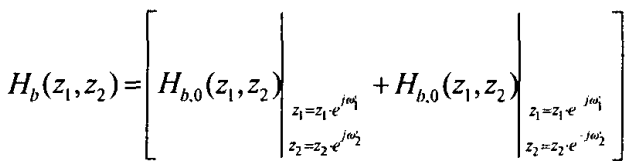

Based on this method, we can found two 2-D BPFs $H_{h, 0}\left(z_{1}, z_{2}\right)$ by applying different $H_{b, 0}\left(z_{1}, z_{2}\right)$ and construct the 2-D NFs by Eq.(2).

First, for

$$
H_{b, 0}(k)=\left(1-\frac{1-z_{1}^{-1}}{1-r_{1} z_{1}^{-1}}\right)\left(1-\frac{1-z_{2}^{-1}}{1-r_{2} z_{22}^{-1}}\right)
$$

we get the 2-D BPF

$H_{b}\left(z_{1}, z_{2}\right)=$

$\frac{2\left(1-r_{1}\right)\left(1-r_{2}\right) z_{1}^{-1} z_{2}^{-1}\left(\cos \left(\omega_{1}+\omega_{2}^{*}\right)-r_{1} \cos \omega_{2}^{-} z_{1}^{-1}-r_{2} \cos \omega_{1} z_{2}^{-1}+r_{1} r_{2} z_{1}^{-1} z_{2}^{-1}\right)}{\left(1-2 r_{1} \cos \omega_{1} z_{1}^{-1}+r_{1}^{2} z_{1}^{-2}\right)\left(1-2 r_{2} \cos \omega_{2}^{-} z_{2}^{-1}+r_{2}^{2} z_{2}^{-2}\right)}$

Form Eq.(2), we get a new 2-D NF (NF2). Next, for

$$
H_{h, 0}\left(z_{1}, z_{2}\right)=\frac{1}{2}\left(1-\frac{r_{1}-z_{1}^{-1}}{1-r_{1} z_{1}^{-1}}\right) \cdot \frac{1}{2}\left(1-\frac{1-z_{2}^{-1}}{1-r_{2} z_{2}^{-1}}\right)
$$

we get another one

$$
\begin{aligned}
& H_{h}\left(z_{1}, z_{2}\right)= \\
& \frac{\left(1-r_{1}\right)\left(1-r_{2}\right)}{2} \cdot \frac{G\left(z_{1}, z_{2}\right)}{\left(1-2 r_{1} \cos \omega_{1} z_{1}^{-1}+r_{1}^{2} z_{1}^{-2}\right)\left(1-2 r_{2} \cos \omega_{2}^{2} z_{2}^{-1}+r_{2}^{2} z_{2}^{-2}\right)}
\end{aligned}
$$

where

$$
\begin{aligned}
& G\left(z_{1}, z_{2}\right)=1+\left(1-r_{1}\right) \cos \omega_{1} z_{1}^{-1}+\left(1-r_{2}\right) \cos \omega_{2}^{-} z_{2}^{-1} \\
& +\left[\left(1+r_{1} r_{2}\right) \cos \left(\omega_{1}^{i}+\omega_{2}^{*}\right)-\left(r_{1}+r_{2}\right) \cos \left(\omega_{1}^{i}-\omega_{2}^{-}\right)\right] z_{1}^{-1} z_{2}^{-1}-r_{1} z_{1}^{-2}-r_{2} z_{2}^{-2} \\
& +r_{1}\left(r_{2}-1\right) \cos \omega_{2}^{-} z_{1}^{-2} z_{2}^{-1}+r_{2}\left(r_{1}-1\right) \cos \omega_{1}^{-} z_{2}^{-2} z_{1}^{-1}+r_{1} r_{2} z_{1}^{-2} z_{2}^{-2}
\end{aligned}
$$

Similarly, we can get another NF (NF3) from Eq.(2).

These two NFs have the same order as NFl and similar denominators. Their block diagram is depicted in Fig. 2 with $F\left(z_{1}, z_{2}\right)=4 \cdot z_{1}^{-1} z_{2}^{-1}\left(\cos \left(\omega_{1}+\omega_{2}\right)-r_{1} \cos \omega_{2} z_{1}^{-1}-r_{2} \cos \omega_{1} z_{2}^{-1}+\right.$ $\left.r_{1} r_{2} z_{1}^{-1} z_{2}{ }^{-1}\right)$ for NF2 and $F\left(z_{1}, z_{2}\right)=G\left(z_{1}, z_{2}\right)$ for NF3.

\section{TWO-DIMENSIONAL ADAPTIVE NOTCH FILTERS}

Since the structures of the NFs proposed in the previous section are much similar to the one proposed in [6], we apply the technique used in the 2-D ANF in [6] here.

First, to guarantee the stability, we implement the denominators of the proposed NFs in lattice form shown in Fig. 3 where the coefficients $(i=1,2)$

$$
a_{i j}(k)=-\cos \left(\hat{\omega_{i}}(k)\right), \quad a_{1 i}(k)=r_{i}^{2}
$$

for NF1 and

$$
a_{0 i}(k)=-\cos \left(\hat{\omega}_{i}(k)\right) \cdot \frac{2 \cdot a_{1 i}(k)}{1+a_{1 i}^{2}(k)}, \quad a_{1 i}(k)=r_{i}^{2}
$$

for NF2 and NF3.

Then the Burg algorithm [7] can be used here as well. Because $a_{11}(k)$ and $a_{12}(k)$ are related to the bandwidths merely, here we only have to use the algorithm to adapt $a_{01}(k)$ and $a_{02}(k)$ with little changes in their update equations. The new algorithm is shown as follows:

Initial condition:

$$
\left(\left(1+r_{i}^{2}\right) \cdot\left|D_{i}(0)\right|>2 r_{i} \cdot\left|C_{i}(0)\right|\right) \quad i=1,2
$$

Main loop: 


$$
\begin{aligned}
& D_{1}(k+1)=\lambda_{1} D_{1}(k)+2 \varepsilon_{1} x_{1}(m-1, n)^{2} \\
& C_{1}(k+1)=\lambda_{1} C_{1}(k)+\varepsilon_{1} x_{1}(m-1, n)\left[x_{1}(m, n)+x_{1}(m-2, n)\right] \\
& a_{01}(k+1)=-\frac{C_{1}(k+1)}{D_{1}(k+1)} \frac{2 r_{1}}{1+r_{1}^{2}} \\
& D_{2}(k+1)=\lambda_{2} D_{2}(k)+2 \varepsilon_{2} x_{2}(m, n-1)^{2} \\
& C_{2}(k+1)=\lambda_{2} C_{2}(k)+\varepsilon_{2} x_{2}(m, n-1)\left[x_{2}(m, n)+x_{2}(m, n-2)\right] \\
& a_{02}(k+1)=-\frac{C_{2}(k+1)}{D_{2}(k+1)} \frac{2 r_{2}}{1+r_{2}^{2}} \\
& a_{0 i}(k+1)=\left\{\begin{array}{cc}
1 & , a_{0 i}(k+1)>1 \\
a_{0 i}(k+1) & , a_{0 i}(k+1) \mid \leq 1 \\
-1 & , a_{0 i}(k+1)<-1
\end{array} \quad i=1,2\right. \\
& \hat{\omega}_{i}(k+1)=\cos ^{-1}\left(-a_{0 i}(k+1)\right) \quad i=1,2 \\
& 0<\lambda_{i}<1 \text { (forgetting factor), } \varepsilon_{i}=1-\lambda_{i} \quad i=1,2
\end{aligned}
$$

By the above algorithm, we can construct $A N F 1, A N F 2$ and $A N F 3$, which represent the adaptive notch filters based on NF1, NF2 and NF3, respectively.

\section{SIMULATION}

Two examples are given in this chapter. First we use the proposed ANF1, ANF2 and ANF3 to eliminate 2-D sinusoidal signals embedded in additive white Gaussian noise (AWGN) and compare the results with the one's given in [6] (ANFO). Next, we display an application of the 2-D ANFs by recovering a contaminated image from 2-D sinusoidal interference.

Example1: Estimation of input frequencies

Assume that the input is given by

$$
u(m, n)=20 \cdot \sin \left(\omega_{i} \cdot m+\omega_{2}^{\prime} \cdot n\right)+v(m, n)
$$

where $(0,0) \leq(m, n) \leq(150,150)$ and $v(m, n)$ is AWGN with zero mean and unit variance. In this example, we set the input frequency (normalized by $2 \pi)$ is $(0.20,0.15)$. We use the proposed ANF1, ANF2 and ANF3 to eliminate the input signal with the settings pole radiuses: $r_{1}=r_{2}=0.95$, the forgetting factors: $\lambda_{1}=\lambda_{2}=0.9999$, initial conditions: $\hat{\omega}_{1}(0)=\hat{\omega}_{2}(0)=\pi / 2$, $D_{1}(0)=D_{2}(0)=0.01, C_{1}(0)=C_{2}(0)=0.005$. Then, we compare their adaptive results with ANF0.

The differences between the results are so slight in visual sight that we only present the results of ANF1 in the following. Fig. 4 is the convergence curves of the coefficients where the upper and lower lines represent $\hat{a_{01}}(k)$ and $\hat{a_{02}}(k)$, respectively. The coefficients finally converged to $a_{01}=-0.3086$ and $a_{02}=-0.5870$, and resulting estimated frequencies are $\omega_{1}=0.40013534 \pi$ and $\hat{\omega_{2}^{\prime}}=0.30029418 \pi$. Fig. $5(\mathrm{a})$ is the output where we see the signal along $n$ time-axis decays faster than $m$ timeaxis because the red line in Fig. 4 converges faster than the blue one. Fig. 5(b) is the magnitude response. The numerical comparison between these NFs is shown in Table 1 where BIAS denotes the estimation error and STD denotes the standard deviation. We see the performances are quite close.

Example 2: Application to image processing

Assume that the input is given by

$$
u(m, n)=50 \sin (0.4 \pi m+0.3 \pi n)+v(m, n)
$$

where $(0,0) \leq(m, n) \leq(255,255)$ and $v(m, n)$ is a pixel of original image, as Fig. 6(a). The image degraded by sinusoidal noise with $\omega_{i}=0.4 \pi$ and $\omega_{2}=0.3 \pi$ is shown in Fig. 6(b). Now let the input to the 2-D adaptive notch filter be $u(m, n)-u$ where

$$
\bar{u}=\frac{1}{256^{2}} \sum_{m=0}^{255} \sum_{n=0}^{255} u(m, n)
$$

If we assume the output is $y(m, n)$, then $y(m, n)+\bar{u}$ shall be the recovered image.

We set $\lambda_{1}=\lambda_{2}=0.9999, r_{1}=r_{2}=0.95$, and all initial conditions are the same as those in Example 1. Using the proposed ANFs, we can get the recovered image in Fig. 6(c) where the sinusoidal noise is gradually eliminated. The coefficients finally converged to $a_{01}=-0.3101$ and $a_{02}=-0.5870$, and resulting estimated frequencies are $\omega_{i}=0.3996521 \pi$ and $\omega_{2}=0.30030439 \pi$. From this example, we see the performance is still good even though the original image is not white.

\section{CONCLUSION}

In this paper, we proposed three new structures for twodimensional adaptive notch filter. The three structures were mainly composed of one all-zero and two 1-D all-pole filters cascaded together. They were simpler than pervious structures. When implementing the two all-pole filter with lattice form, we can apply an algorithm similar to the one proposed in [6]. In addition, the estimated notch frequencies were found from the two 1-D filters separately. Simulation result demonstrated the validity of the proposed 2-D adaptive notch filter.

\section{REFERENCES}

[1] N. I. Cho, C. H. Choi and S. U. Lee, "Adaptive line enhancement by using an IIR lattice Notch filter", IEEE Trans. ASSP, vol.37, no.4, pp.585-589, Aprl. 1989.

[2] P. A. Regalia, "An improved lattice-based adaptive IIR Notch filter", IEEE Trans. SP, vol.39, no.9, pp.2124-2128, Sept. 1991.

[3] S. C. Pei and C. C. Tseng, "Two dimensional IIR digital Notch filter design", IEEE Trans. Circuits Syst. II, vol. 41, no.3, pp.227-231, Mar. 1997. 
[4] S. C. Pei and C. C. Tseng, "Corrections to two dimensional IIR digital Notch filter design", IEEE Trans. Circuit Syst. II, vol.41, no.9, pp.630, Sept. 1994.

[5] S. C. Pei, W. S. Lu and C. C. Tseng, "Analytical twodimensional IIR Notch filter design using outer product expansion", IEEE Trans. Circuits Syst. II, vol.44, no.9, pp.765-768, Sept. 1997.

[6] T. Hinamoto, N. Ikeda, S. Nishimura and A. Doi, "Design of two dimensional adaptive digital Notch filters", Proceedings of ICSP2000, pp.538-542.

[7] N. I. Cho, C. H. Choi and S. U. Lee, "Adaptive line enhancement by using an IIR lattice Notch filter", IEEE Trans ASSP, vol.37, np.4, pp.585-589, April 1989.

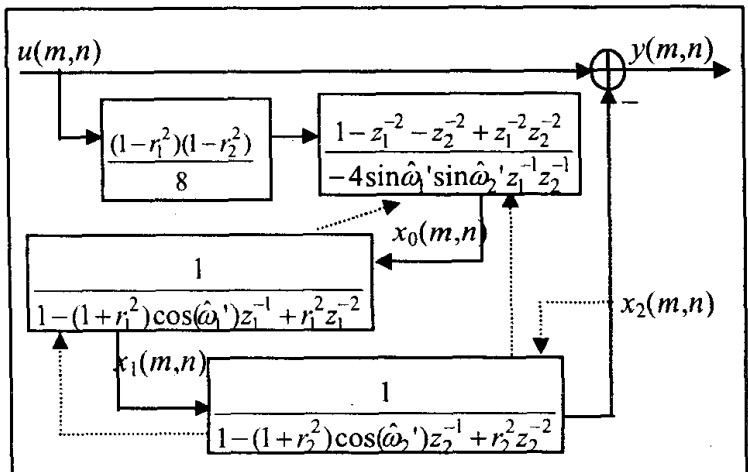

Fig. 1. Block diagram of NF1

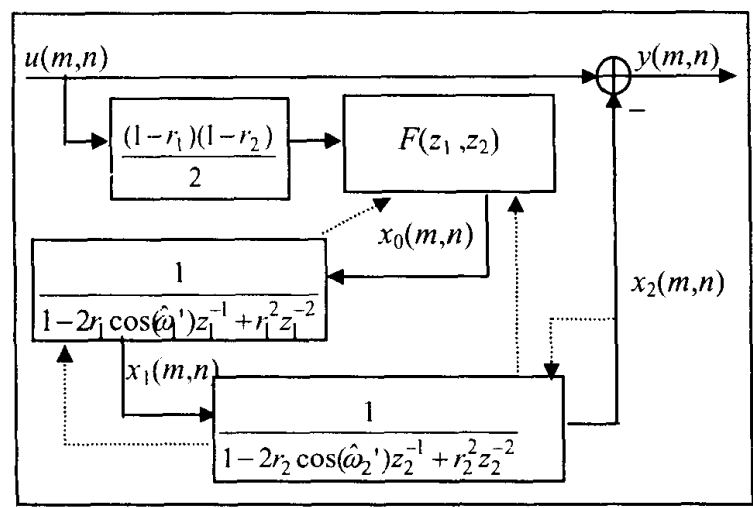

Fig. 2. Block diagram of NF2 or NF3

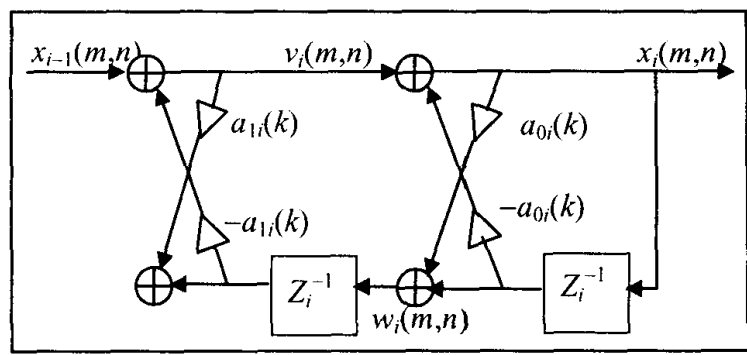

Fig. 3. A variable IIR filter

\begin{tabular}{|c|c|c|c|c|}
\hline & BIAS1 & BIAS2 & STD1 & STD2 \\
\hline ANF0 & $-1.5808 \mathrm{e}-004$ & $-1.8367 \mathrm{e}-005$ & $4.6970 \mathrm{e}-006$ & $2.3783 \mathrm{e}-006$ \\
\hline ANF1 & $-2.0804 \mathrm{e}-005$ & $1.4015 \mathrm{e}-004$ & $3.2487 \mathrm{e}-006$ & $2.1935 \mathrm{e}-006$ \\
\hline ANF2 & $-1.2543 \mathrm{e}-004$ & $-1.2087 \mathrm{e}-005$ & $2.7791 \mathrm{e}-006$ & $2.2496 \mathrm{e}-006$ \\
\hline AMF3 & $-8.9263 \mathrm{e}-005$ & $-1.1628 \mathrm{e}-005$ & $3.0736 \mathrm{e}-006$ & $1.8559 \mathrm{e}-006$ \\
\hline
\end{tabular}

Table 1. Performance Comparison between the ANFs

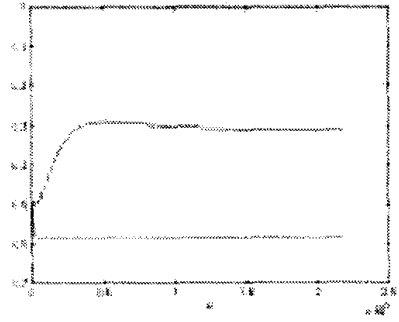

Fig. 4. Convergence of the coefficients of NF1
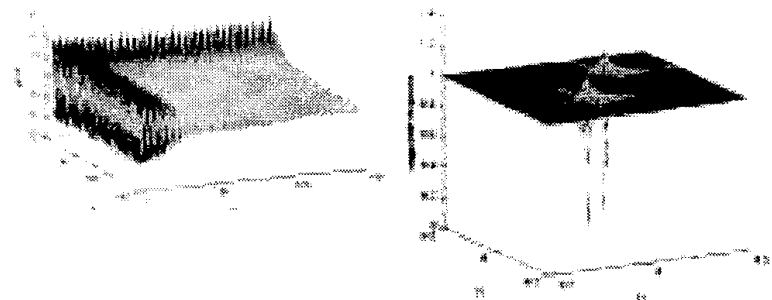

(a)

(b)

Fig. 5. (a)The output of NF1 (b) The magnitude response of NF1
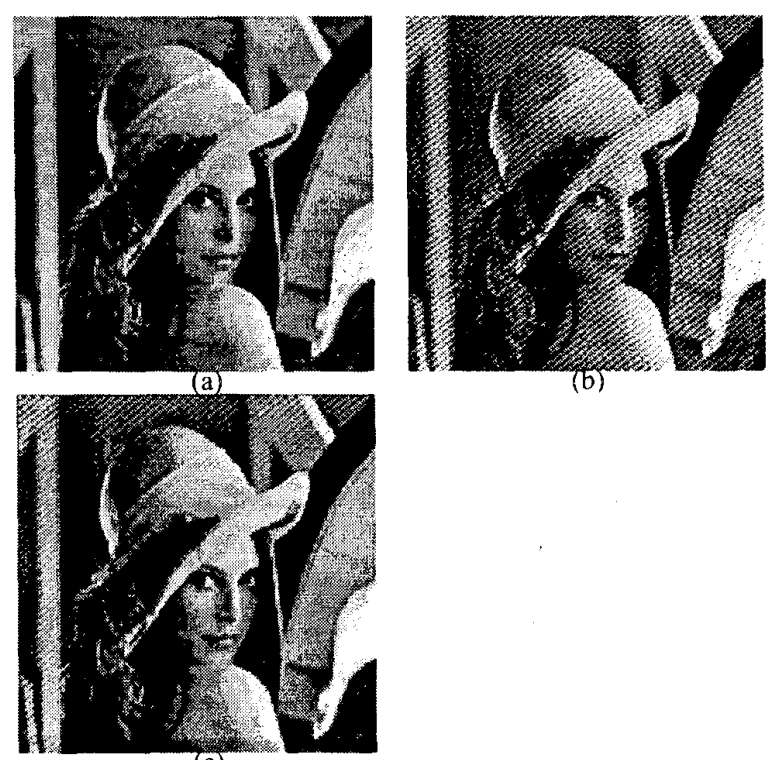

(b)

(c)

Fig. 6. Application of the 2-D ANF (a) the original image, (b) the degraded image $(c)$ the recovered image. 